\title{
Surveying the Nucleon-Nucleon Momentum Correlation Function in the Framework of Quantum Molecular Dynamics Model
}

\author{
Y. G. Ma* Y. B. Wei, W. Q. Shen, X. Z. Cai, J. G. Chen, J. H. Chen, D. Q. Fang, W. Guo, \\ C. W. Ma, G. L. Ma, Q. M. Su, W. D. Tian, K. Wang, T. Z. Yan, C. Zhong, and J. X. Zuo \\ Shanghai Institute of Applied Physics, Chinese Academy of Sciences, P.O. Box 800-204, Shanghai 201800, China
}

(Dated: August 7, 2018)

\begin{abstract}
Momentum correlation functions of the nucleon-nucleon pairs are presented for reactions with $\mathrm{C}$ isotopes bombarding a ${ }^{12} \mathrm{C}$ target within the framework of the isospin-dependent quantum molecular dynamics model. The binding-energy dependence of the momentum correlation functions is also explored, and other factors that have an influence on momentum correlation functions are investigated. These factors include momentum-dependent nuclear equation of state, in-medium nucleon-nucleon cross sections, impact parameters, total pair momenta, and beam energy. In particular, the rise and the fall of the strength of momentum correlation functions at lower relative momentum are shown with an increase in beam energy.
\end{abstract}

PACS numbers: 25.10.+s, 25.70.Mn, 21.45.+v, 27.20.+n

\section{INTRODUCTION}

The method of two-particle intensity interferometry was developed by Hanbury-Brown and Twiss [1] in the early 1950s. Originally they applied two photon correlation to measure the angular diameter of stars and other astronomical objects. Initially, the method did not receive universal acceptance before a number of terrestrial experiments were performed to confirm it. Now the method of intensity interferometry is commonly referred as the Hanbury-Brown/Twiss (HBT) effect. Although the original application of the HBT effect used photons as the detected particles, it was rapidly realized that the approach can be generalized to include correlation measurements for other bosons and fermions as well. The first measurements of the HBT effect in subatomic physics came from elementary-particle reactions. Goldhaber et al. extracted the spatial extent of the annihilation fireball in proton-antiproton reactions from two-pion correlations [2]. In fact, the method explores that identical particles situated nearby in phase-space experience quantum statistical effects resulting from the (anti)symmetrization of the multi-particle wave function. For Bosons, therefore, the two-particle coincidence rate shows an enhancement at small momentum difference between the particles. The momentum range of this enhancement can be related to the size of the particle source in coordinate space. Recently, there are not only substantial experimental literatures on the technical applications, but also a large number of theoretical papers on momentum correlations with different models from low energy to relativistic energy heavy ion collisions. For reviews, see the references [3, 4, 5, 6]. More recently, HBT method has been extended to others fields, for instance, the analogous corre-

*Email: ygma@sinap.ac.cn lations in semiconductors and in free space aiming at the fermionic statistics of electrons [7, 8].

Many experimental measurements of HBT effect have been performed for heavy ion collisions (HIC) at intermediate energy in recent years. With the increasing of beam energies, nucleon-nucleon collision plays a dominate role during the reaction process in the intermediate energy, which results in an increasing importance of earlier particle emission. By the application of the two-particle correlation function, one could obtain the information on particle emission and collision dynamics.

In this energy domain, most correlation measurements focus on two-proton correlation functions. The shape of a two-proton correlation function reflects the combined effects of the Pauli blocking principle, Coulomb interaction and proton-proton $(p-p)$ nuclear interaction. Earlier measurements of two-proton correlation functions in intermediate energy heavy ion collisions performed by Lynch et al. 9 provided the evidence for particle emission from localized highly-excited regions. After that, many experimental groups have investigated the momentum correlation functions in various aspects, such as for the unstable particle populations [10], the dependence on the impact parameter 11], the dependence of the total momentum of nucleon pairs 12], the dependence of the isospin of the emitting source 13 and so on. More interestingly, HBT technique has been used to construct neutron-neutron correlation function which is useful to investigate the properties of neutron-halo nuclei 14, 15, 16]. The details about the nuclear Equation of State (EOS) and the collision dynamics could be revealed from the correlation function by the comparison between the experimental data and the transport model calculations.

However, in most studies of $p-p$ correlation functions, the HBT strength at $20 \mathrm{MeV} / c$ of the proton-proton relative momentum is taken a unique quantity to determine the source size and/or emission time of two proton 
emission. In a recent analysis for the HBT data below $100 \mathrm{MeV} /$ nucleon with the imaging method, Verde et al. show that the width of correlation function provides the information of the source size of the fast dynamical component while the peak of the correlation function is sensitive to relative yield from slow and fast emission components [17, 18]. In addition, it was claimed that the proton emission from slow statistical component was not suitably treated in the conventional transport model, such as BUU model [19]. In light of the above studies, the whole shape of the correlation function is important for deducing space-time information of the emission source [17]. For heavy ion reactions with mid-heavy projectile and target combination in the Fermi energy domain, the slow emission component should not be neglected. To minimize the complication of the slow and fast component of proton emission in correlation function, it might be useful to choose light reaction systems at higher beam energies. In this context, we will use $\mathrm{C}+{ }^{12} \mathrm{C}$ system to investigate momentum correlation functions above 100 $\mathrm{MeV} /$ nucleon in this work.

To understand the details of collisions for different reactions by the HBT studies, a reliable simulation of the collision dynamical process for the heavy ion reaction is required. The simulation shall give a reasonable treatment of fragment formation after the final state interaction. There are some good event-generator models to describe the collision process. In relativistic heavy ion collisions, successful models include both the stringhadronic-like models, such as relativistic quantum molecular dynamics and the parton cascade model etc. In intermediate energy region, the successful transport model includes BUU model [20] and Quantum Molecular Dynamics (QMD) model 21]. From those event-generator models one can obtain the phase space of the emitted particles with different parameters of the EOS and then construct the momentum correlation function. Recently, the nuclear symmetry energy dependence of HBT has been also explored through the isospin dependent BUU model 22] in intermediate energy heavy ion collisions. Moreover, the nuclear binding energy and separation energy dependences of the HBT strength have been investigated with the help of the Isospin Dependent Quantum Molecular Dynamics model (IDQMD) 23, 24]. In this paper, more features of momentum correlation function are reported by using the IDQMD model.

The paper is organized as follows: in Sec. II we describe the HBT technique and the IDQMD model. The stability of the IDQMD model is checked; Section III presents the results and discussions. We discuss the influences of the following ingredients: initialization of projectile and target, emission time of nucleons, evolution time of the reaction, gate on the total momentum of nucleonnucleon pair, soft and stiff momentum dependent EOS, in-medium nucleon-nucleon cross section, impact parameter and incident energy etc. Finally the conclusions are presented in Section IV.

\section{HBT TECHNIQUE AND THE IDQMD MODEL}

Firstly, we recall the HBT technique. As we know, the wave function of relative motion of light identical particles is modified by the final-state interaction and quantum statistical symmetries when they are emitted in close proximity in space-time, and this is the principle of intensity interferometry, i.e. HBT. In standard KooninPratt formalism [25, 26, 27], the two-particle correlation function is obtained by convoluting the emission function $g(\mathbf{p}, x)$, i.e., the probability for emitting a particle with momentum $\mathbf{p}$ from the space-time point $x=(\mathbf{r}, t)$, with the relative wave function of two particles,

$$
C(\mathbf{P}, \mathbf{q})=\frac{\int d^{4} x_{1} d^{4} x_{2} g\left(\mathbf{P} / 2, x_{1}\right) g\left(\mathbf{P} / 2, x_{2}\right)|\phi(\mathbf{q}, \mathbf{r})|^{2}}{\int d^{4} x_{1} g\left(\mathbf{P} / 2, x_{1}\right) \int d^{4} x_{2} g\left(\mathbf{P} / 2, x_{2}\right)}
$$

where $\mathbf{P}\left(=\mathbf{p}_{\mathbf{1}}+\mathbf{p}_{\mathbf{2}}\right)$ and $\mathbf{q}\left(=\frac{1}{2}\left(\mathbf{p}_{\mathbf{1}}-\mathbf{p}_{\mathbf{2}}\right)\right)$ are the total and relative momenta of the particle pair respectively, and $\phi(\mathbf{q}, \mathbf{r})$ is the relative two-particle wave function with their relative position $\mathbf{r}=\left(\mathbf{r}_{2}-\mathbf{r}_{1}\right)-\frac{1}{2}\left(\mathbf{v}_{\mathbf{1}}+\mathbf{v}_{\mathbf{2}}\right)\left(t_{2}-t_{1}\right)$. This approach is very useful in studying effects of nuclear equation of state and nucleon-nucleon cross sections on the reaction dynamics of intermediate energy heavy-ion collisions [5].

In the viewpoint of theoretical simulation, the correlation function can be established by using an event generator that produces the phase space information by modelling the collision dynamics and particle production. The event-generator correlation functions are then constructed from the positions and momenta representing the single-particle emission distribution at the time of the last strong interaction, i.e. at freeze-out. In the present work, the event-generator is the Isospin-dependent Quantum Molecular Dynamics transport model [21], which has been successfully applied to the HBT studies of the heavy-ion collisions for neutron-rich nuclei induced reactions 23, 24]. Using a computation code named CRAB (Correlation After Burner ) of Pratt 28], which takes into account final-state nucleon-nucleon interactions, we have evaluated two-nucleon correlation functions from the emission function given by the IDQMD model. In the following part, we shall introduce the model briefly.

The Quantum Molecular Dynamics approach is a many body theory to describe heavy ion reactions from intermediate energy to $2 \mathrm{GeV} /$ nucleon 29]. It includes several important ingredients: initialization of the target and the projectile; nucleon propagation in the effective potential; nucleon-nucleon collisions in nuclear medium; Pauli blocking effect and the numerical test. A general review about the QMD model can be found in 21]. The IDQMD model is based on the QMD model affiliating the isospin factors.

The heavy-ion collision dynamics at intermediate energies is mainly governed by three components: the mean field, two-body collisions and Pauli blocking. Therefore, for an isospin-dependent reaction dynamics model it is 
important to affiliate isospin degrees of freedom with the above three components. In addition, the sampling of phase space of neutrons and protons in the initialization should be treated separately because of larger difference between neutron and proton density distributions for nuclei far from the $\beta$-stability line. For exotic neutron-rich nucleus one should sample a stable initialized nucleus with neutron-skin or halo structure so that one can directly incorporate nuclear structure effects into a microscopic transport process. The IDQMD model has been improved based on the above ideas and the details will be given in the following.

In the present calculations the interaction potential in the IDQMD is determined as follows:

$$
U(\rho)=U^{\mathrm{Sky}}+V^{\mathrm{Coul}}+U^{\mathrm{sym}}+V^{\mathrm{Yuk}}+U^{\mathrm{MDI}}+U^{\text {Pauli }},
$$

where $U^{\text {Sky }}$ is the density-dependent Skyrme potential and it reads when the momentum dependent potential is included

$$
U^{\text {Sky }}=\alpha\left(\frac{\rho}{\rho_{0}}\right)+\beta\left(\frac{\rho}{\rho_{0}}\right)^{\gamma}+t_{4} \ln ^{2}\left[\varepsilon\left(\frac{\rho}{\rho_{0}}\right)^{2 / 3}+1\right] \frac{\rho}{\rho_{0}},
$$

where $\rho$ and $\rho_{0}$ are total nucleon density and its normal value, respectively. The parameters $\alpha, \beta, \gamma, t_{4}$ and $\varepsilon$ are related to the nuclear equation of state and listed in Table. 1. $V_{c}$ is Coulomb potential. $U^{\text {Yuk }}$ is the Yukawa potential ,

$$
U^{\text {Yuk }}=t_{3} \frac{\exp \left(\frac{\left|\overrightarrow{r_{1}}-\overrightarrow{r_{2}}\right|}{m}\right)}{\frac{\left|\overrightarrow{r_{1}}-\overrightarrow{r_{2}}\right|}{m}}
$$

where $m=0.8 \mathrm{fm}$. $U^{\mathrm{MDI}}$ is the momentum dependent interaction [29],

$$
U^{\mathrm{MDI}}=t_{4} \ln ^{2}\left[t_{5}\left(\overrightarrow{p_{1}}-\overrightarrow{p_{2}}\right)^{2}+1\right] \frac{\rho}{\rho_{0}}
$$

where $\overrightarrow{p_{1}}$ and $\overrightarrow{p_{2}}$ are the momentum of two interacting nucleons. $U^{\text {Pauli }}$ is the Pauli potential,

$$
U^{\text {Pauli }}=V_{p}\left(\frac{\hbar}{p_{0} q_{0}}\right)^{3} \exp \left(-\frac{\left(\overrightarrow{r_{i}}-\overrightarrow{r_{j}}\right)^{2}}{2 q_{0}^{2}}-\frac{\left(\overrightarrow{p_{i}}-\overrightarrow{p_{j}}\right)^{2}}{2 p_{0}^{2}}\right) \delta_{p_{i} p_{j}}
$$

where

$$
\delta_{p_{i} p_{j}}= \begin{cases}1 & \text { for neutron-neutron or proton-proton } \\ 0 & \text { for neutron-proton. }\end{cases}
$$

The parameters $V_{p}, p_{0}$ and $q_{0}$ is $30 \mathrm{MeV}, 400 \mathrm{MeV} / c$ and $5.64 \mathrm{fm}$, respectively. $U^{\text {sym }}$ is the symmetry potential. In the present calculation, we use $U^{\mathrm{sym}}=C_{\mathrm{sym}} \frac{\left(\rho_{n}-\rho_{p}\right)}{\rho_{0}} \tau_{z}$ where $C_{\text {sym }}$ is the strength of symmetry potential, taking the value of $32 \mathrm{MeV} . \rho_{n}$ and $\rho_{p}$ are the neutron density and the proton density, respectively. $\tau_{z}$ is the $z$ th component of the isospin degree of freedom, which equals 1 or -1 for neutrons or protons, respectively. The parameters of the interaction potential are given in Table. 1 where $K=200$ or $380 \mathrm{MeV}$ means the soft- or the stiffmomentum dependent potential, respectively.

\begin{tabular}{|c|c|c|c|c|c|c|c|}
\hline$\alpha$ & $\beta$ & $\gamma$ & $t_{3}$ & $t_{4}$ & $t_{5}$ & $\varepsilon$ & $K$ \\
\hline$(\mathrm{MeV})$ & $(\mathrm{MeV})$ & & $(\mathrm{MeV})$ & $(\mathrm{MeV})$ & $\left(\mathrm{MeV}^{-2}\right)$ & $(\mathrm{MeV})$ & $(\mathrm{MeV})$ \\
\hline-390.1 & 320.3 & 1.14 & 7.5 & 1.57 & $5 \times 10^{-4}$ & 21.54 & 200 \\
\hline-129.2 & 59.4 & 2.09 & 7.5 & 1.57 & $5 \times 10^{-4}$ & 21.54 & 380 \\
\hline
\end{tabular}

Table 1. The parameters of the interaction potentials

The in-medium nucleon-nucleon (NN) cross section can be parameterized as isospin dependent from the available experimental data. Studies of collective flow in HIC at intermediate energies revealed the reduction of the inmedium NN cross sections 30, 31, 32]. An empirical expression of the in-medium NN cross section [31] is used:

$$
\sigma_{\mathrm{NN}}^{\mathrm{med}}=\left(1+f \frac{\rho}{\rho_{0}}\right) \sigma_{\mathrm{NN}}^{\text {free }}
$$

with the factor $f \approx-0.2$ which has been found to better reproduce the flow data [30]. Here $\sigma_{\mathrm{NN}}^{\text {free }}$ is the experimental NN cross section 33. The free neutron-proton cross section $\sigma_{\mathrm{NN}}^{\text {free }}$ is about a factor of 3 times larger than the free neutron-neutron or proton-proton cross section below about $400 \mathrm{MeV} /$ nucleon in the laboratory energy. It should be mentioned that the relationship between the neutron-proton cross section and neutron-neutron (proton-proton) cross section depends also on the modification of the nuclear density distributions during the reactions.

The Pauli blocking effect in IDQMD model is treated separately for the neutron and the proton: Whenever a collision occurs, we assume that each nucleon occupies a six-dimensional sphere with a volume of $\hbar^{3} / 2$ in the phase space (considering the spin degree of freedom), and then calculate the phase volume, $V$, of the scattered nucleons being occupied by the rest nucleons with the same isospin as that of the scattered ones. We then compare $2 \mathrm{~V} / \hbar^{3}$ with a random number and decide whether the collision is blocked or not.

When the initialization of the projectile and the target is taken in the IDQMD, the density distributions of protons and neutrons are distinguished from each other. The references of neutron and proton density distributions for the initial projectile and target nuclei in IDQMD are taken from the Skyrme-Hartree-Fock (SHF) method with parameter set SKM*. Using this density distribution, we could get the initial coordinate of nucleons in nuclei in terms of the Monte-Carlo sampling method. The momentum distribution of nucleons is generated by means of the local Fermi gas approximation:

$$
P_{F}^{i}(\vec{r})=\hbar\left(3 \pi^{2} \rho_{i}(\vec{r})\right)^{\frac{1}{3}},(i=n, p) .
$$

In the model, the radial density can be written as:

$$
\begin{aligned}
\rho(r)= & \sum_{i} \frac{1}{(2 \pi L)^{3 / 2}} \exp \left(-\frac{r^{2}+r_{i}^{2}}{2 L}\right) \frac{L}{2 r r_{i}} \\
& \times\left[\exp \left(\frac{r r_{i}}{L}\right)-\exp \left(-\frac{r r_{i}}{L}\right)\right]
\end{aligned}
$$


where $L$ is the so-called Gaussian wave width (here $L=$ $\left.2.16 \mathrm{fm}^{2}\right)$.

Stability of the initialized nucleus has been checked by the time evolution of the system at zero temperature 24]. The accepted configurations are quite stable: only a few percentage nucleons escape from the nucleus till $200 \mathrm{fm} / c$ in the intermediate energy domain. In addition, the stability is also checked by tracking the time evolutions of the average binding energy and the root mean square radius of the initialized nucleus and good enough stability has been found. Lighter nuclei are somewhat less stable. One or two out of ten nuclei lose a nucleon in the required time span. To avoid taking unstable initialization of projectile and target in the IDQMD calculation, we only select the initialization samples of nuclei which meet the required stability.

Nuclear fragments are constructed by a modified isospin-dependent coalescence model, in which particles with relative momentum smaller than $p_{0}=300 \mathrm{MeV} / c$ and relative distance smaller than $R_{0}=3.5 \mathrm{fm}$ will be combined into a cluster.

In our calculations, the reactions of C-isotopes with ${ }^{12} \mathrm{C}$ have been performed. Most simulations are done in head-on collisions $(b=0 \mathrm{fm})$ at $100 \mathrm{MeV} /$ nucleon or 800 $\mathrm{MeV} /$ nucleon. The momentum correlation function are constructed by the phase space points at $200 \mathrm{fm} / c$ when the system is basically at the freeze-out stage.

\section{RESULTS AND DISCUSSIONS}

\section{A. Stable initialization vs random initialization}

Fig. 1]shows proton-neutron correlation functions from the reactions induced by a chain of $\mathrm{C}$-isotopes projectile on ${ }^{12} \mathrm{C}$ target at $800 \mathrm{MeV} /$ nucleon of incident energy and head-on collisions (impact parameter $b=0 \mathrm{fm}$ ) when the suitable selection of the stable initialization is used. In this figure the HBT strength of each isotope in lower relative momentum can be separated. If we plot this strength at $5 \mathrm{MeV} / c\left(C_{p-n}\right)$ as a function of the mean binding energy $\left(E_{b}\right)$ of the projectiles (C-isotopes), we find that there exists an approximate linear relationship between $C_{p-n}$ and $E_{b}$ as it is shown by the solid circles in Fig. 2

In order to investigate the importance of initialization in the HBT study, a comparison has been performed using a random initialization for the projectile and the target in the IDQMD based on Monte-Carlo sampling. In random initialization, the phase space of the nucleons is generated with Monte Carlo random sampling. During sampling of each nucleon, if the distance between every two nucleons is larger than $1.5 \mathrm{fm}$ and the product of the space radius and the momentum radius between two nucleons is larger than one constant according to the uncertain relationship, the sampling will be accepted. In this case, there is no additional requirement of the binding energy of the projectile or the target.

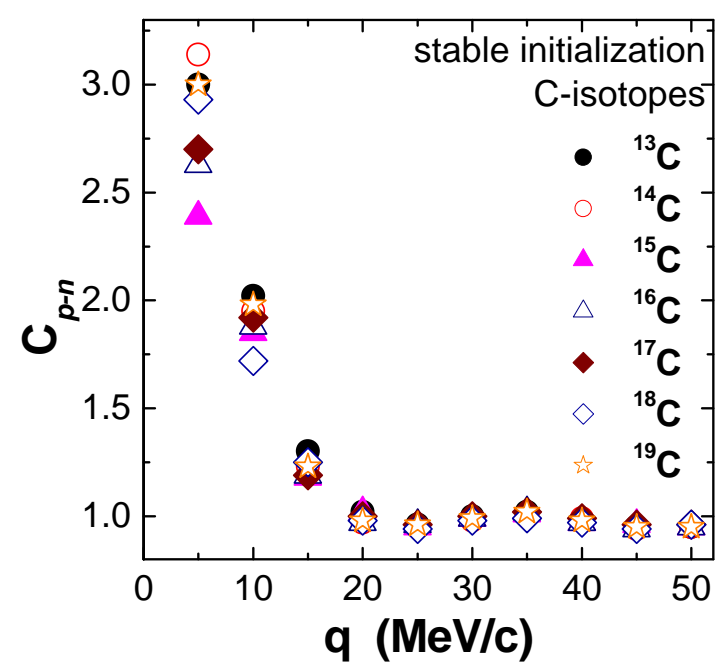

FIG. 1: (Color online) The proton-neutron correlation function $C_{p-n}$ for the reactions of a chain of C-isotopes with ${ }^{12} \mathrm{C}$ at $800 \mathrm{MeV} /$ nucleon and $b=0 \mathrm{fm}$ using the stable initialization of projectile and target. The meaning of symbols are illustrated in the insert.

Taken such a random initialization, the initial phase space of the nucleons for the projectile and the target is different event by event. Through the transport process of IDQMD, we can obtain the momentum correlation function and extract the strength of HBT in the final states. Fig. 3 shows the neutron-proton HBT for different $\mathrm{C}$-isotopes by random sampled initialization. Similar to Fig. 11 the strength at $5 \mathrm{MeV} / c$ is not a constant for different isotopes induced reactions which is shown by open circles in Fig. 2 However, two apparent differences can be observed between the results of stable initialization and random initialization. One is the difference in the magnitude of the HBT strength. The values of $C_{p-n}$ at $5 \mathrm{MeV} / c$ with the random initialization are less than those with the stable initialization. For the random initialization, the initial phase space which may not meet the requirement of the ground state as required in SHF calculation fluctuates event by event. In this case the tightness of initial nucleus becomes weaker than that with stable initialization of phase space which is sampled by the SHF density distribution. Thus, the values of $C_{p-n}$ at $5 \mathrm{MeV} / c$ which can embody the tightness between the nucleons become smaller. The other difference is the slope of $C_{p-n}$ vs $E_{b}$. A steeper linear relationship is observed for the stable initial phase space, while the dependence of $C_{p-n}$ vs $E_{b}$ becomes weaker in the random case.

The comparison between both different initialization methods indicates that the reasonable initial phase space of the projectile which is sampled by the experimental $E_{b}$ and SHF density calculation in the IDQMD is important and suitable to investigate the dependence of the bind- 


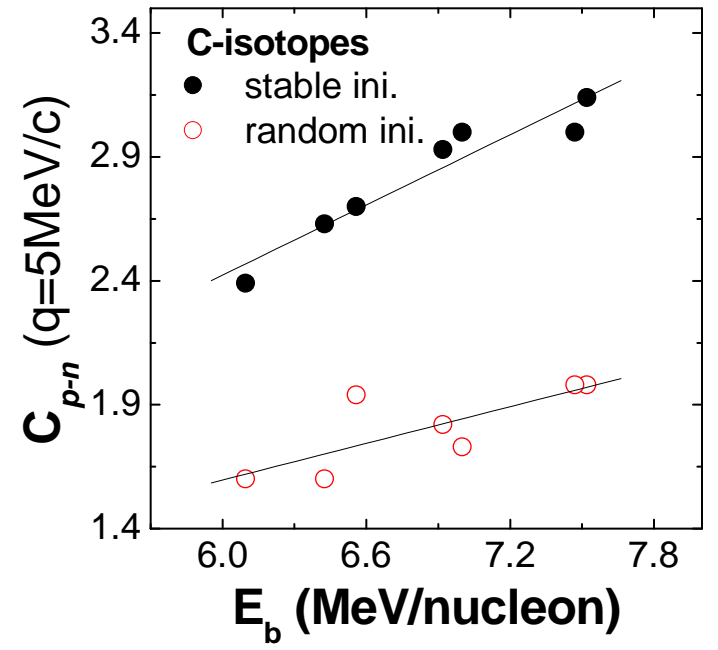

FIG. 2: (Color online) The relationship between the protonneutron correlation function $C_{p-n}$ at $5 \mathrm{MeV} / c$ and the average binding energy per nucleon of $\mathrm{C}$-isotopes. The filled circles represent the results with the stable initial phase space taking in the IDQMD model according to the SHF density distribution. The open circles represent the results with the random initial phase space. The collisions were simulated at $800 \mathrm{MeV} /$ nucleon and $b=0 \mathrm{fm}$. The target is ${ }^{12} \mathrm{C}$. The lines are linear fits to guide the eyes.

ing energy for some observables. In the following calculations, we will use the stable initialization to study some features of momentum correlation functions.

\section{B. Emission time of nucleons and evolution time of reaction}

In the intermediate energy domain, the extraction of the space-time information is further complicated by two effects. One is the presence of multiple sources of particle emission [34], another is the different time scale of statistical and dynamical emission from equilibrium and non-equilibrium sources [17, 18]. The total momentumgated correlation function can be used to investigate the later effects. Understanding the emission time sequence of neutrons and protons will be helpful to understand the nuclear interaction. It also might be sensitive to the nuclear equation of state. It has been shown that the emission times of the nucleons are related to their kinetic energies. Generally, earlier emitted nucleons have higher energies than later ones. Some results have been demonstrated in the experiments [35].

Fig. 4 shows the time evolution of the particle emission rate by using the stiff momentum-dependent (Stiff M) EOS. The open circles connected with solid line show the proton emission rate and the filled ones represent the neutron emission rate. The collisions are simulated for ${ }^{18} \mathrm{C}+{ }^{12} \mathrm{C}$ at $100 \mathrm{MeV} /$ nucleon and $b=0 \mathrm{fm}$. In the fol-

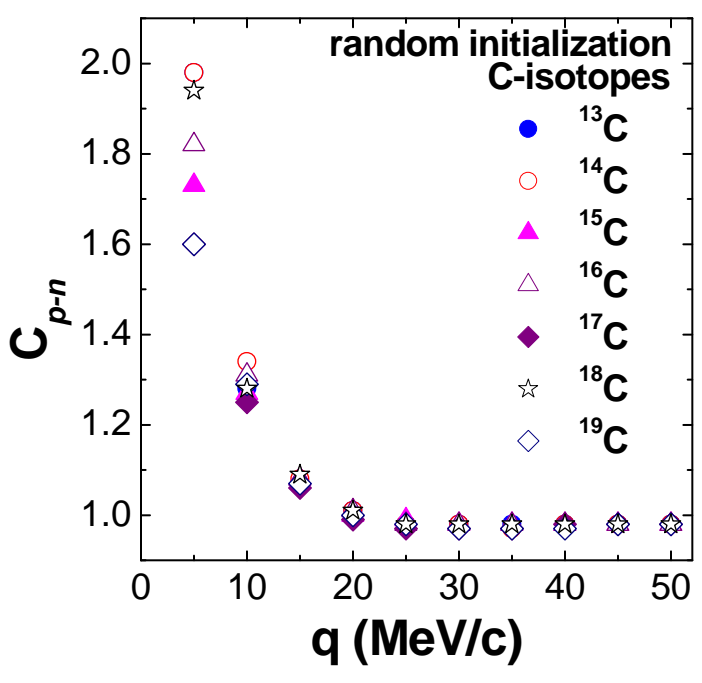

FIG. 3: (Color online) The proton-neutron correlation function $C_{p-n}$ for the reactions of a chain of C-isotopes with ${ }^{12} \mathrm{C}$ target at $800 \mathrm{MeV} /$ nucleon using the random initialization of the projectile and the target. Symbols which represent the different isotopes are illustrated in the insert.

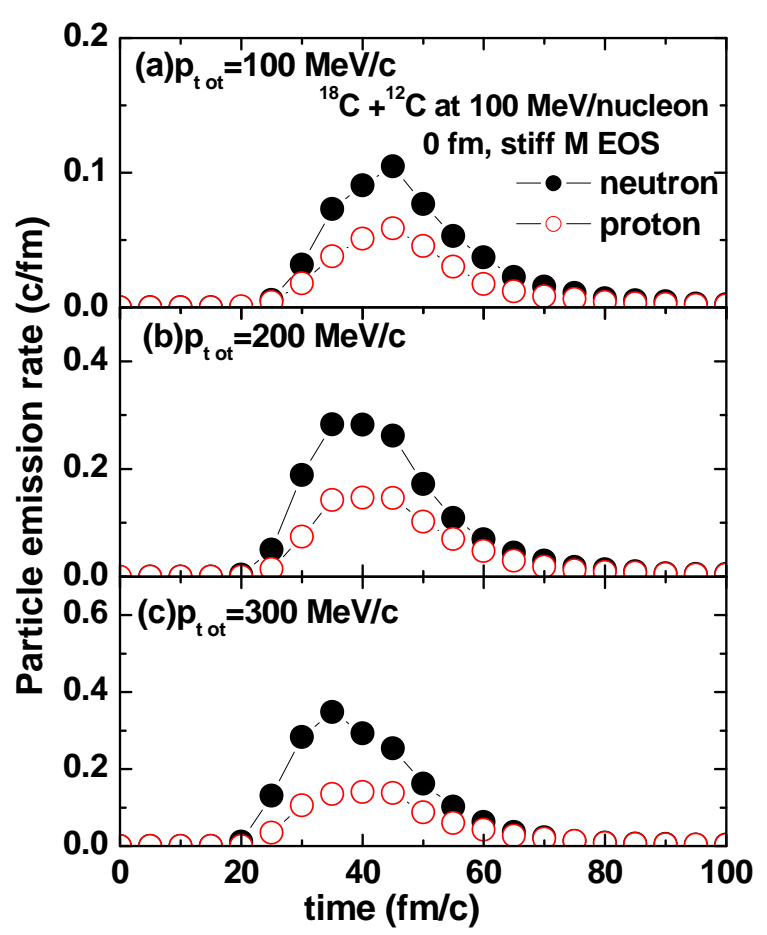

FIG. 4: (Color online) The time evolution of the emission rate of nucleons for ${ }^{18} \mathrm{C}+\mathrm{C}$ at $100 \mathrm{MeV} /$ nucleon and $b=0 \mathrm{fm}$ with the different gates of the total nucleon-nucleon pair momentum. 
lowing discussions, except the special note, the collisions are all performed in the above circumstance.

From this figure, nucleons begin to be emitted around $20 \mathrm{fm} / c$ and their emission rates reach to their maximum 15-20 fm/c later. With increasing total nucleon-nucleon momentum $P_{\text {tot }}$, peak of the emission rate of nucleons becomes larger and its corresponding time tends to earlier time. This indicates that nucleons with higher total pair momentum are emitted earlier. Higher momentum nucleons mostly belong to pre-equilibrium emission nucleons and essentially originate from higher density regions. In contrast, lower momentum nucleons are mostly emitted from equilibrium-like sources. For neutron-proton pairs with lower total momentum, the emission rate of neutron and of proton is almost synchronous, ie. there is no obvious difference of the emission sequence between neutron and proton. However, for the neutron-proton pair with larger total momentum, the emission rate of neutrons reaches to the peak value earlier than that of protons, it means that, on average, neutrons are emitted earlier than protons. The reason why the emission rate of neutrons is larger than that of protons stems from the neutron-rich content of projectile. Two interpretations seem to be possible. On one hand, the symmetry potential term in Eq.(2) plays an important role in controlling the emission of nucleons. In neutron-rich projectile induced reaction, protons could feel stronger attractive potential due to neighboring neutrons which results in more bound protons for disassembling sources. Viceversa, neutrons will, on one side, feel the stronger repulsive interaction due to more neutron-neutron pairs and, on the other side, feel smaller attractive potential because of the decreasing of the average assortative number of nearest neighboring protons for a certain neutron for increasing isospin of the source. Both reasons will lead to produce more unbound neutrons for disassembling sources with higher isospin [36].

Experimentally, the momentum correlation function reflects the information in the final state of the reaction. Theoretically, the final state can be seen as the state at the freeze-out of system. Since the HBT is sensitive to the space-time information, we shall investigate the HBT at different evolution times of reaction. Fig. 5 shows the correlation functions of neutron-neutron, proton-neutron and proton-proton when the evolution time of reaction $t$ $=150,200,250,300$ and $400 \mathrm{fm} / c$. Generally, HBT values become smaller in later evolution time due to the weakness of the nucleon-nucleon correlation when the system is diluted. However, we can roughly say that the HBT values do not change dramatically after $t=$ $200 \mathrm{fm} / c$ after $t=200 \mathrm{fm} / c$ is compared to earlier times. Because of the limited computation resource, thereafter we investigate the features of HBT for all systems when $t=200 \mathrm{fm} / c$.

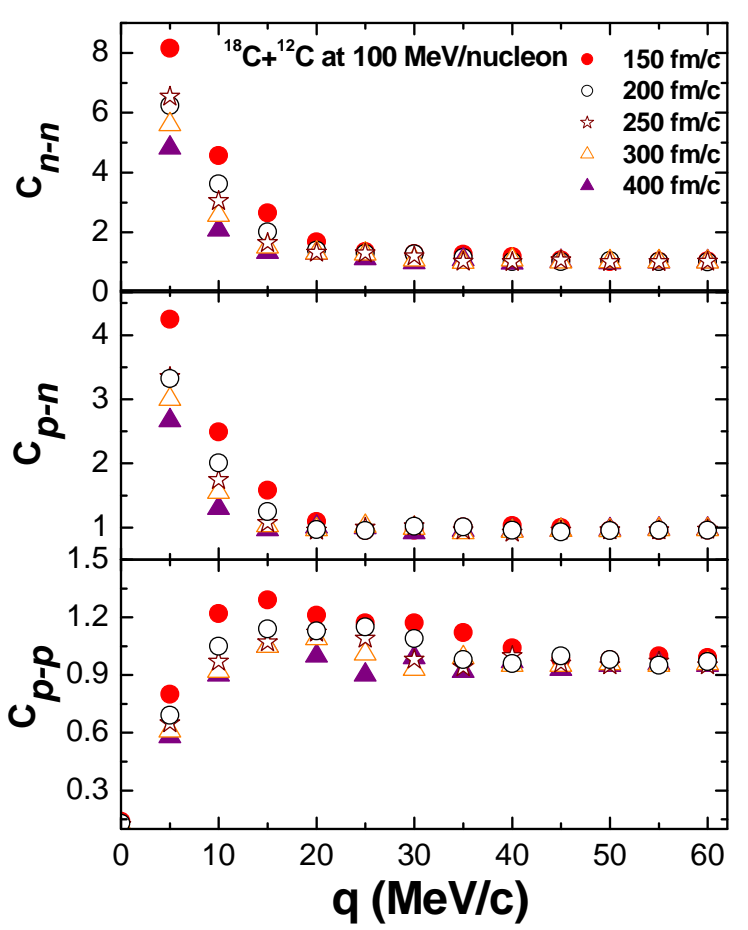

FIG. 5: (Color online) The momentum correlation functions of $\mathrm{n}-\mathrm{n}, \mathrm{p}-\mathrm{n}$ and p-p pairs for ${ }^{18} \mathrm{C}+\mathrm{C}$ at $100 \mathrm{MeV} /$ nucleon and $b=0$ $\mathrm{fm}$ are constructed in different evolution time of the system. The evolution time is illustrated in the insert.

\section{Gate of the total momentum}

Since the magnitude of the total pair momentum is related to the nucleon emission time, we shall discuss the effect of the total pair momentum on HBT in this section. Earlier emission time induces stronger correlation, larger total momentum thus contributes to the strength of correlation function too. We discuss the calculations with different total nucleon-nucleon pair momentum ( $\left.P_{\text {tot }}\right)$ in the following. The results are shown in Fig. 6. In this figure, three types of nucleon-nucleon correlation function, namely that of n-n, n-p and p-p, are shown. From the left panels, it is cleanly observed that the higher the $P_{\text {tot }}$, the higher the HBT value in lower relative momentum. While, from the right panels, it is observed that HBT value in the region of lower relative momentum is higher with the soft EOS than that with the stiff EOS.

To see an overall trend of strength of HBT versus $P_{\text {tot }}$, we plot the value of $C_{n-n}\left(C_{p-n}\right)$ at $5 \mathrm{MeV} / c$ or $C_{p-p}$ at $20 \mathrm{MeV} / c$ as a function of $P_{\text {tot }}$ in Fig. [7 The filled circles connected with the solid line present the results with the soft momentum-dependent EOS and the open circles connected with the solid line show the ones with the stiff momentum-dependent EOS.

From the figure, it is clear that the strength of twonucleon correlation function is smaller at lower total pair momentum than that of the higher one. As it is shown 


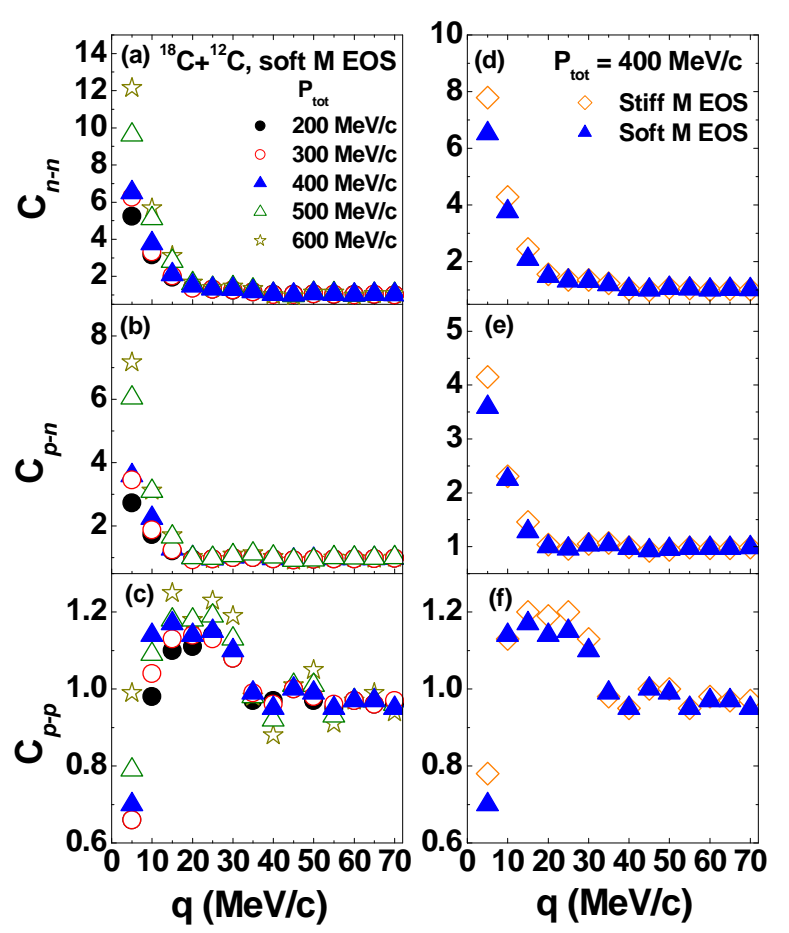

FIG. 6: (Color online) The two-nucleon correlation function $C_{n-n}$, $C_{p-n}$ and $C_{p-p}$ for ${ }^{18} \mathrm{C}+\mathrm{C}$ at $100 \mathrm{MeV} /$ nucleon and $b=0 \mathrm{fm}$ with the different total pair momentum $\left(P_{\text {tot }}\right)$ cuts or EOS parameters: the left panels correspond to the different gates of $P_{\text {tot }}$ while the right panels correspond to different EOS for $P_{\text {tot }}=400 \mathrm{MeV} / c$.

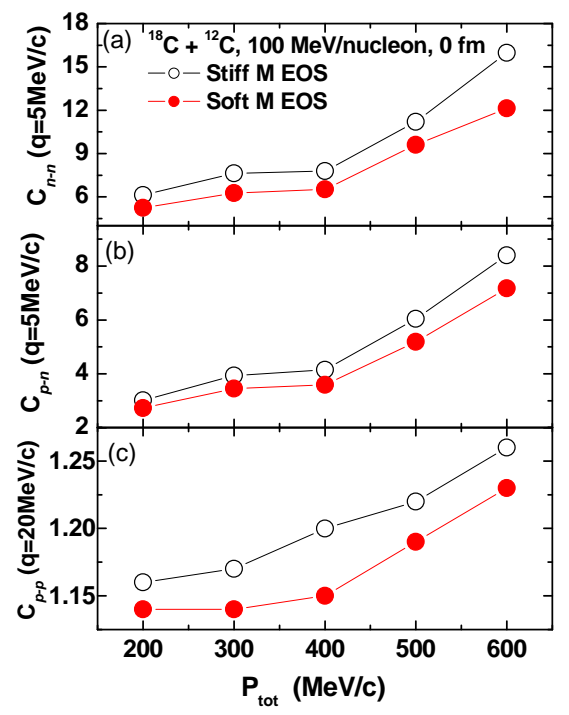

FIG. 7: (Color online) The HBT strength of two-nucleon correlation function for n-n and p-n at $5 \mathrm{MeV} / c$ or at p-p at $20 \mathrm{MeV} / c$ for ${ }^{18} \mathrm{C}+\mathrm{C}$ at $100 \mathrm{MeV} /$ nucleon as a function of the gate of the total momentum $\left(P_{\text {tot }}\right)$ of the nucleon-nucleon pairs in the collisions.

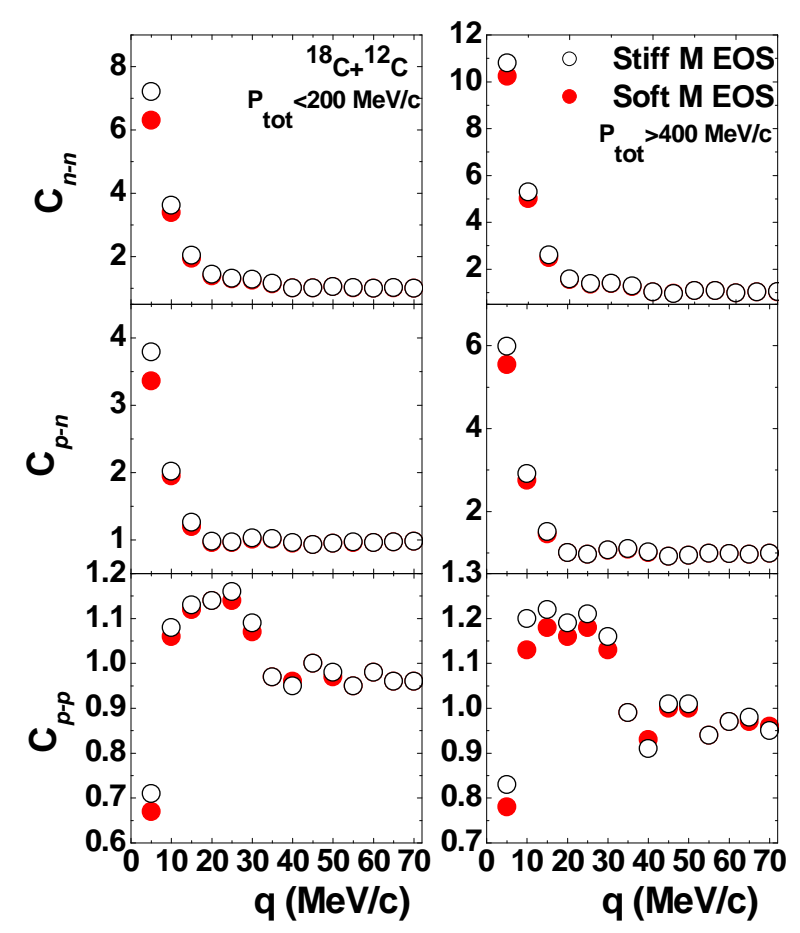

FIG. 8: (Color online) The comparison between the correlation function with soft momentum-dependent (Soft $\mathrm{M}$ ) and stiff momentum-dependent (Stiff M) EOS for ${ }^{18} \mathrm{C}+\mathrm{C}$ at 100 $\mathrm{MeV} /$ nucleon and $b=0 \mathrm{fm}$ in IDQMD calculations. The left panels correspond to the cases of $P_{\text {tot }}<200 \mathrm{MeV} / c$ while the right panels correspond to the cases of $P_{\text {tot }}>400 \mathrm{MeV} / c$. The filled and open circles represent the results with Soft- and Stiff- M EOS, respectively.

the nucleons with lower total momenta are emitted later than those with higher total momenta which naturally reduces the HBT strength. The calculated difference indicates the qualitative characterization of the emission process during the collisions. On the other hand, the tendency is similar despite the different combination of nucleon-nucleon pair. Experimental results for the momentum-gated nucleon pairs show a trend similar to the one that can be found in the literature, see for example Ref. [11, 12].

In addition, one can find that the HBT strength with the stiff momentum-dependent EOS is higher than that with the soft one. The influence of the different momentum dependent EOS on HBT will be discussed in the next section in details.

\section{Soft and stiff momentum-dependent potential}

The EOS is considered an important property of nuclear matter and several researches have been performed to investigate on the EOS of the finite nuclear matter [37]. In this section, we will show the results of correlation functions with different EOS parameters.

In previous studies, the role of different potentials, 
i.e, the soft and stiff potential in the transport models, has been investigated via some physical observables. However, in most case, the potential does not include the momentum dependent term, eg. in HBT studies with isospin dependent BUU [22]. In this work we used a momentum-dependent part in the potential, namely the soft momentum-dependent potential (Soft M EOS) and the stiff momentum-dependent potential (Stiff M EOS). we use also an isospin-dependent potential and calculated nucleon-nucleon correlation functions with the above mentioned two types of momentum-dependent potentials. The results are shown in Fig. 8

In Fig. 8 the filled circles connected with solid line represent the correlation function with Soft M EOS and the open ones are the results with the one with Stiff M EOS. The left parts are the HBT results with $P_{\text {tot }}<$ $200 \mathrm{MeV} / c$ and the right parts are the results $P_{\text {tot }}>$ $400 \mathrm{MeV} / c$. From the figure, it is clear that the correlation function with the stiff potential is larger than that with the soft potential, which is similar to the calculation results with BUU 11]. Stiff potential makes the compression of nucleonic matter difficult compared with the case with the soft one and leads to larger emission rate and earlier average emission time of nucleons, which leads to stronger correlation function for the stiff potential.

\section{E. In-medium nucleon-nucleon cross section}

The effect of the in-medium nucleon-nucleon cross section $\left(\sigma_{\mathrm{NN}}^{\mathrm{med}}\right)$ is discussed in this section. We use a value of $\sigma_{\mathrm{NN}}^{\text {med }}$ which is different from the free nucleon-nucleon cross section $\left(\sigma_{\mathrm{NN}}^{\text {free }}\right)$ to investigate its influence on momentum correlation functions. Fig. 9 shows NN, NP and PP correlation functions for different $\sigma_{\mathrm{NN}}^{\text {med }}$ and EOS. Slightly larger values of the HBT is found for larger $\sigma_{\mathrm{NN}}^{\text {med }}$, especially for $0.8 \sigma_{\mathrm{NN}}^{\text {free }}$, as well as for the stiff EOS. Fig. 10 shows the HBT strengths at $5 \mathrm{MeV} / c$ (for NN and NP) or at $20 \mathrm{MeV} / c$ (for PP) as a function of $\sigma_{\mathrm{NN}}^{\text {med }}$ in the stiff and soft EOS. The strength increases with $\sigma_{\mathrm{NN}}^{\text {med }}$ and with the stiffness of the EOS. This can be understood by the following arguments: with the increasing of the in-medium nucleon-nucleon cross section, the collision rate between two nucleons increases and consequently the system reaches to the equilibrium stage faster. Before equilibrium is reached, more pre-equilibrium nucleons are emitted, which makes the strength of the correlation function larger.

\section{F. Impact parameter dependence}

Considering the importance of the Wigner function which depends on the impact parameter, we shall investigate on momentum correlation function at different impact parameter. Collisions of ${ }^{18} \mathrm{C}+{ }^{12} \mathrm{C}$ are performed at $100 \mathrm{MeV} /$ nucleon and at the impact parameters of 0 , $1,2,3,4$ and $5 \mathrm{fm}$. The calculated total momentum inte-

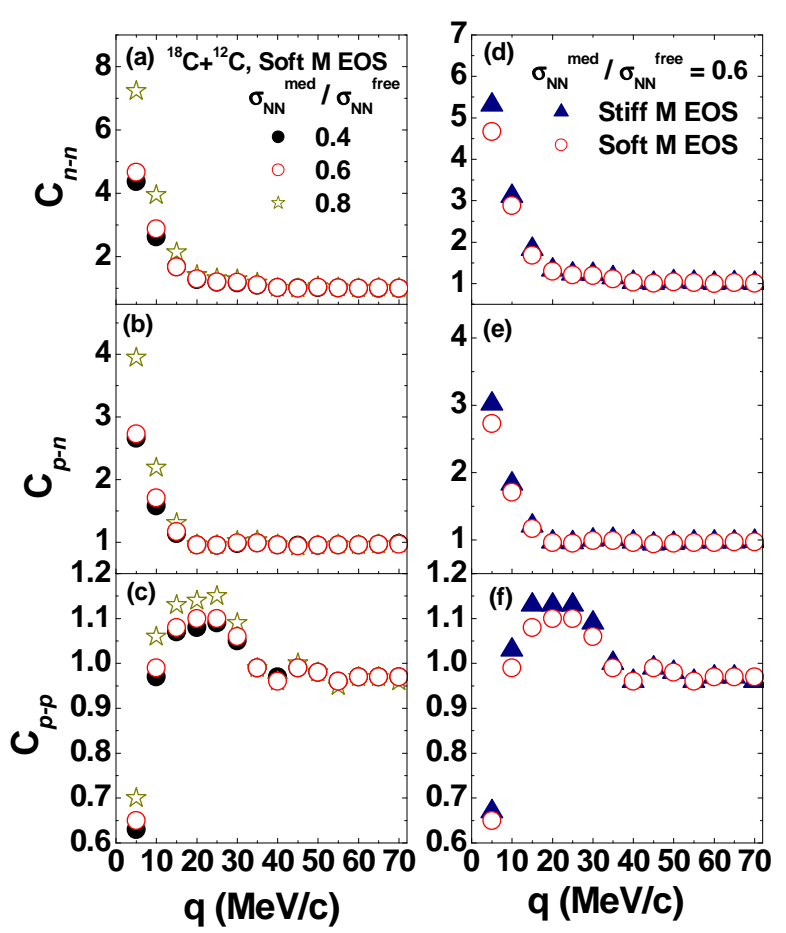

FIG. 9: (Color online) The two-nucleon correlation function $C_{\mathrm{NN}}$, $C_{p-n}$ and $C_{p-p}$ for ${ }^{18} \mathrm{C}+\mathrm{C}$ at $100 \mathrm{MeV} /$ nucleon and $b=0 \mathrm{fm}$ with different in-medium nucleon-nucleon cross section $\left(\sigma_{\mathrm{NN}}^{\text {med }} / \sigma_{\mathrm{NN}}^{\text {free }}\right)$ (left panels) or different EOS (right panels) for fixed $0.6 \sigma_{\mathrm{NN}}^{\text {free }}$.

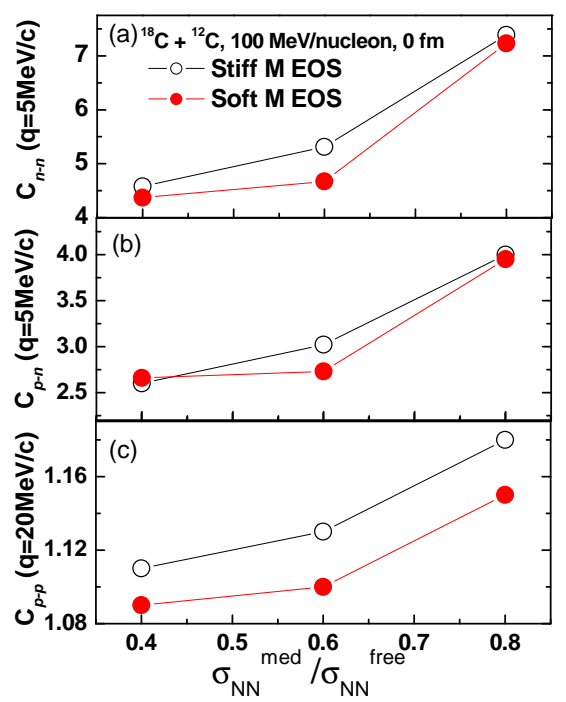

FIG. 10: (Color online) The HBT strengths of NN (a) and PN (b) at $5 \mathrm{MeV} / c$ and $\mathrm{PP}$ (c) at $20 \mathrm{MeV} / c$ as a function of the in-medium nucleon-nucleon cross section $\left(\sigma_{\mathrm{NN}}^{\text {med }} / \sigma_{\mathrm{NN}}^{\text {free }}\right)$ from the reaction ${ }^{18} \mathrm{C}$ $+{ }^{12} \mathrm{C}$ at $100 \mathrm{MeV} /$ nucleon and $b=0 \mathrm{fm}$. The open and filled symbols represent stiff and soft momentum-dependent potential, respectively. 

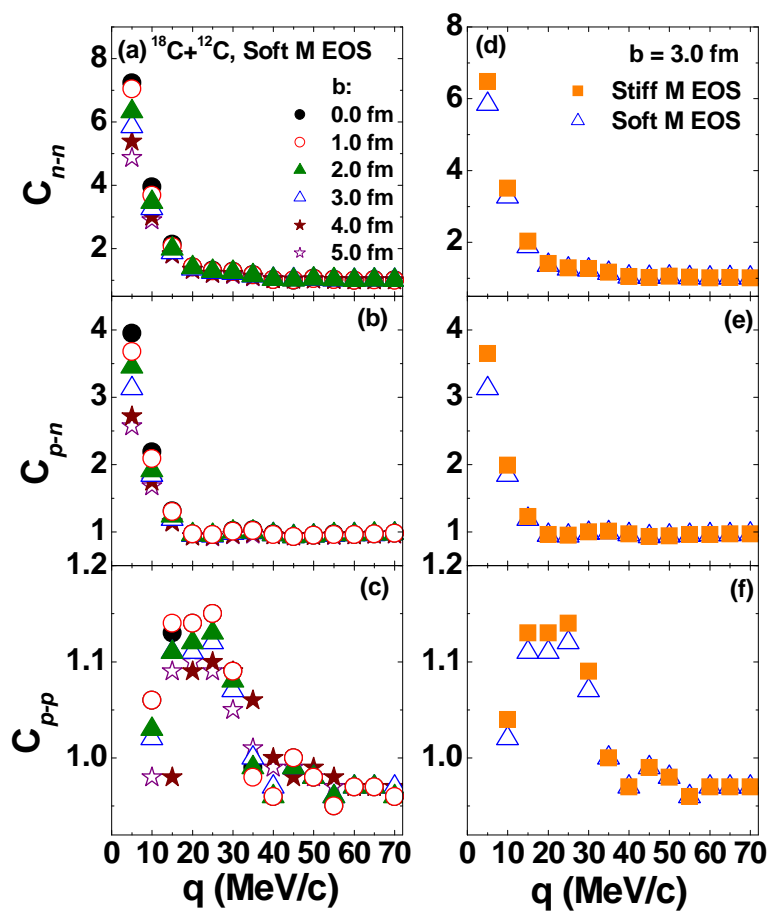

FIG. 11: (Color online) Momentum correlation functions for $\mathrm{n}-$ $\mathrm{n}, \mathrm{p}-\mathrm{n}$ and $\mathrm{p}-\mathrm{p}$ are calculated in different impact parameters (left panels) or with different EOS at fixed $b=3 \mathrm{fm}$ for ${ }^{18} \mathrm{C}+\mathrm{C}$ at 100 $\mathrm{MeV} /$ nucleon.

grated correlation functions are shown in Fig. 11] Larger HBT values for central collisions or stiff EOS are predicted. Their strengths are shown in Fig. 12

In Ref. 11], some explanations on the effect of impact parameter have been presented. As we already know, the strength of the correlation function mainly depends on the emission time and the source size. From the figure, there exists a large difference between soft and stiff potentials. Secondly, the strength of correlation function becomes weaker with increasing impact parameter on both soft and stiff momentum-dependent EOS. This indicates that the stiffness of the potential in the IDQMD does not change the tendency of the HBT strength of HBT with increasing impact parameter. On the other hand, the behavior of the HBT strength with impact parameter might reflect the changed size of the emitting source. In central collisions, nucleon-nucleon collisions are very frequent and emitted nucleons are mostly from one compact and hot dense region. Therefore, the HBT strength is larger because of the smaller source size, if compared to peripheral collisions. Also some other reasons including Fermi jets may contribute to this effect [38].

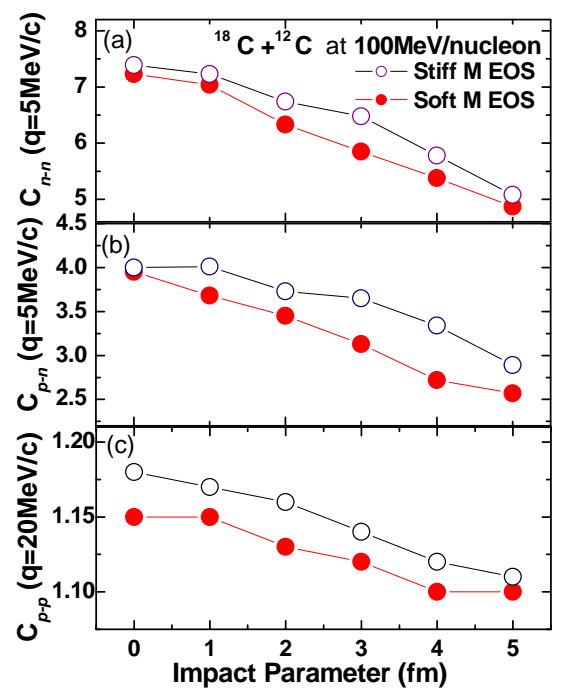

FIG. 12: (Color online) The strength of the nucleon-nucleon correlation functions of $\mathrm{n}-\mathrm{n}$ (a) and $\mathrm{NP}$ (b) at $5 \mathrm{MeV} / c$ or PP (C) at $20 \mathrm{MeV} / c$ for ${ }^{18} \mathrm{C}+\mathrm{C}$ at $100 \mathrm{MeV} /$ nucleon as a function of impact parameter. The open and filled symbols represent stiff and soft momentum-dependent potential, respectively.

\section{G. Incident energy dependence}

The influence of the incident energy on HBT is investigated in this section. Fig. 13 shows the momentum correlation function at different beam energies (left panels) and different EOS (right panels). Fig. 14 displays the calculated HBT strengths of n-n (a), p-n (b) and p-p (c) as a function of beam energy for head-on collisions. From the figure, both stiff and soft momentum potential provides a similar evolution of the HBT strength with beam energy. Interestingly, the HBT strength first increases with the incident energy and reaches a peak around 100 $\mathrm{MeV} /$ nucleon and then decreases at higher incident energies. The raise of the HBT strength at lower incident energies can be essentially attributed to drastic nucleonnucleon collision at higher energies, which results, on average, in earlier emission of nucleons. However, the decreasing behavior of HBT strengths with incident energies higher than $100 \mathrm{MeV} /$ nucleon cannot be explained in this way.

In order to discuss the possible origin of the complex behavior of the HBT strength with incident energy, we investigate the maximum nucleon emission rate in the whole time evolution as a function of beam energy in Fig. 15] From the figure, the maximum nucleon emission rates show a rise and fall behavior similar to the one observed in Fig. 15 for the HBT strength. Around $100 \mathrm{MeV} /$ nucleon, the nucleon emission rate is maximum. This energy coincides with the peak position of the HBT strength in Fig. [15. From this point of view, the HBT strength could be correlated to the maximum nucleon emission rate during heavy ion collisions. The 

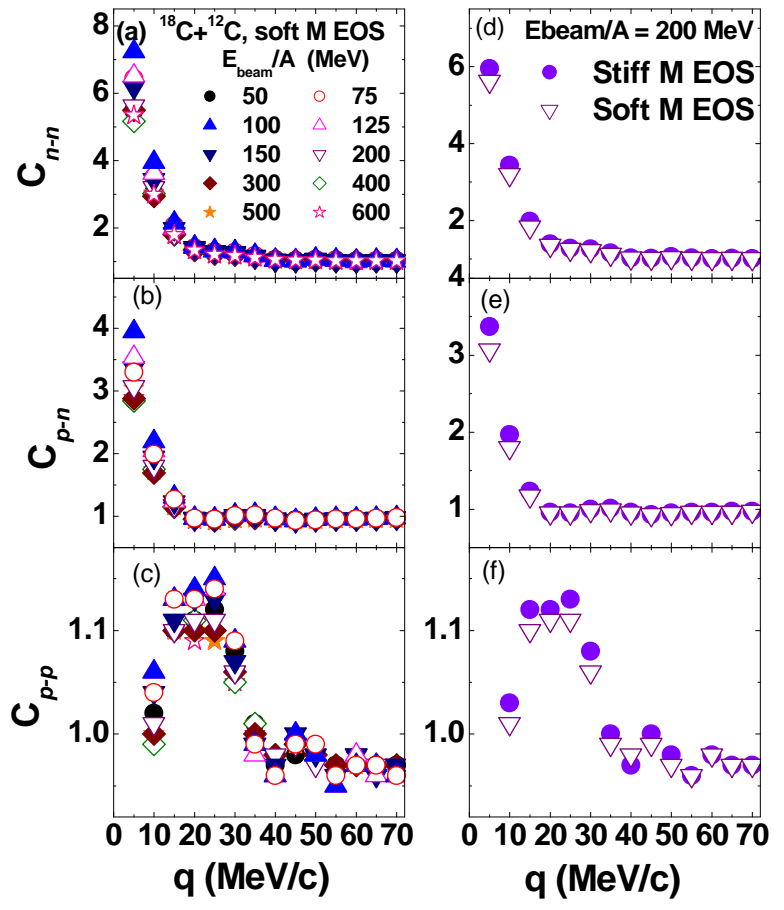

FIG. 13: (Color online) The momentum correlation function of $n-n$ (a), p-n (b) and p-p (c) pairs in different beam energies in head-on collisions for soft M EOS (left panels) or different EOS at $E_{\text {beam }}$ $=200 \mathrm{MeV} /$ nucleon (right panels).

higher the nucleon emission rate, the stronger the HBT strength. Of course, another possible explanation for the falling branch of HBT strength can be attributed to the source size in the final state $(t=200 \mathrm{fm} / c)$. It is expected that in the energy range of a few hundreds of $\mathrm{MeV} /$ nucleon, where the repulsive nucleon-nucleon interaction is dominant, a more dilute system might develop after freeze-out. So the fall of HBT with beam energy above $100 \mathrm{MeV} /$ nucleon indicates that the source size has been much expanded in the final state at higher energies. In contrast, the attractive mean field competes with the repulsive nucleon-nucleon interaction below 100 $\mathrm{MeV} /$ nucleon. Shorter emission times with increasing beam energies may play a dominant role in determining the behavior of the HBT strength. Fig. [15]also illustrates illustrates that the emission rate of neutrons is larger than that of protons and that the neutrons are emitted earlier than protons.

\section{SUMMARY}

In summary, isospin-dependent quantum dynamics model has been used as an event-generator to study the momentum correlation function of neutron-neutron, proton-proton and neutron-proton correlation functions for $\mathrm{C}+{ }^{12} \mathrm{C}$. An approximate linear relationship between

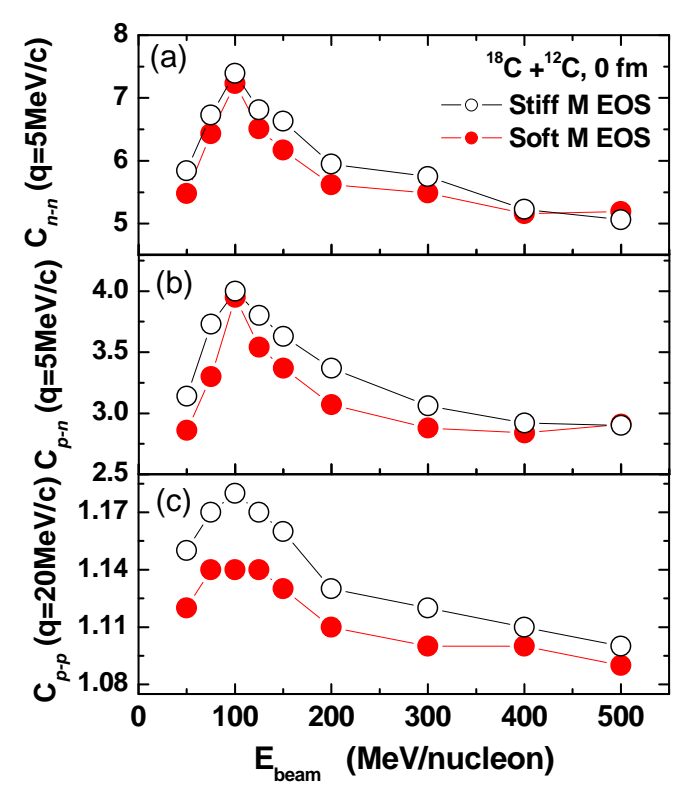

FIG. 14: (Color online) HBT strengths of n-n (a), p-n (b) and p-p (c) as a function of beam energy in head-on collisions. Open or filled circles represent the calculations with stiff or soft momentum potential, respectively.

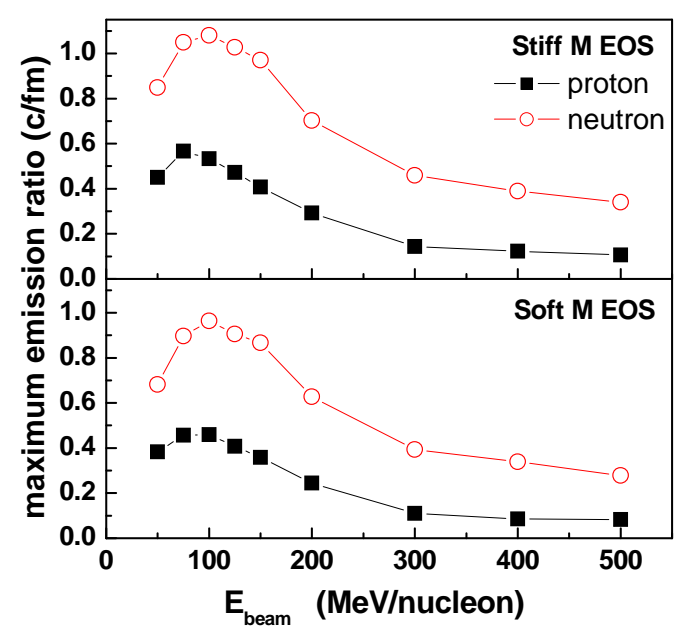

FIG. 15: (Color online) The maximum nucleon emission rate as a function of the incident energy. Squares and circles represent proton and neutron, respectively.

the strength of correlation function at low relative momentum and the mean binding energy of projectiles has been revealed and the influence of the different initialization methods of the projectiles on momentum correlation function has been also investigated. In addition, some different physical ingredients which have effects on momentum correlation functions have been shown in this work. These ingredients include nuclear equation of state and in-medium nucleon-nucleon cross section. It shows 
that the stiff momentum-dependent EOS or a larger inmedium nucleon-nucleon cross section results in stronger HBT strength than the soft momentum-dependent EOS or a smaller in-medium nucleon-nucleon cross section. Some other aspects which have also effects on momentum correlation function, including the time evolution of the reaction system, the impact parameter, the gate of the total momenta of nucleon-nucleon pairs and the incident energy are also explored. Results show that the HBT strength decreases with increasing impact parameter due to the earlier emission or a compact source size in central collisions. A positive correlation between the gate of total nucleon-nucleon pair momentum and the HBT strength can be explained by the earlier emission time of nucleons with higher total momentum. The rise and fall of the HBT strengths with the beam energy can be interpreted either in terms of the maximum nucleon emission rates during heavy-ion collisions or in terms of the dominant shorter emission time with increasing beam energy below $100 \mathrm{MeV} /$ nucleon and the larger later-stage expansion of the source size with the increasing of beam energy above $100 \mathrm{MeV} /$ nucleon.

\section{Acknowledgments}

This work was supported in part by the Shanghai Development Foundation for Science and Technology under Grant Numbers 05XD14021 and 03 QA 14066, the National Natural Science Foundation of China under Grant No 10328259, 10135030, 10535010 the Major State Basic Research Development Program under Contract No G200077404.
[1] R. Hanbury Brown, R.Q. Twiss, Nature 178, 1046 (1956).

[2] G. Goldhaber, S. Goldhaber, W. Lee, and A. Pais, Phys. Rev. 120, 300 (1960).

[3] U. Heinz and B. Jacak, Ann. Rev. Nucl. Part. Sci. 49, 529 (1999).

[4] D. H. Boal, C. K. Gelbke, B. K. Jennings, Rev. Mod. Phys. 62, 553 (1990).

[5] W. Bauer, C. K. Gelbke, S. Pratt, Ann. Rev. Nucl. Part. Sci. 42, 77 (1992).

[6] U. A. Wiedemann and U. Heinz, Phys. Rep. 319, 145 (1999).

[7] M. Henny, S. Oberholzer, C. Strunk, T. Heinzel, K. Ensslin, M. Holland, and C. Schönenberger, Science 284, 296 (1999); William D. Oliver, Jungsang Kim, Robert C. Liu, and Yoshihisa Yamamoto, Science 284, 299 (1999).

[8] R. de-Picciotto, M. Reznikov, M. Heiblum, V. Umansky, G. Bunin, D. Mahalu, Nature 389, 162 (1997).

[9] W. G. Lynch, C. B. Chitwood, M. B.Tsang, D. J. Fields, D. R. Klesch, C. K. Gelbke, G. R. Young, T. C. Awes, R. L. Ferguson, F. E. Obenshain, F. Plasil, R. L. Robinson, and A. D. Panagiotou, Phys. Rev. Lett. 51, 1850 (1983).

[10] J. Pochodzalla, C. K. Gelbke, W. G. Lynch, M. Maier, D. Ardouin, H. Delagrange, H. Doubre, C. Grégoire, A. Kyanowski, W. Mittig, A. Péghaire, J. Péter, F. SaintLaurent, B. Zwieglinski, G. Bizard, F. Lefébvres, B. Tamain, J. Qubert, Y. P. Viyogi, W. A. Friedman, D. H. Boal, Phys. Rev. C 35, 1695 (1987).

[11] W. G. Gong, W. Bauer, C.K. Gelbke, S. Pratt, Phys. Rev. C 43, 781 (1991).

[12] N. Colonna, D.R. Bowman, L. Celano, G. D'Erasmo, E. M. Fiore, L. Fiore, A. Pantaleo, V. Paticchio, G. Tagliente, and S. Pratt, Phys. Rev. Lett. 75, 4190 (1995).

[13] R. Ghetti, V. Avdeichikov, B. Jakobsson, P. Golubev, J. Helgesson, N. Colonna, G. Tagliente, H.W. Wilschut, S. Kopecky, V.L. Kravchuk, E.W. Anderson, P. NadelTuronski, L. Westerberg, V. Bellini, M.L. Sperduto, C. Sutera, Phys.Rev. C 69, 031605 (2004).

[14] N. A. Orr, Nucl. Phys. A 616, 155 (1997).

[15] F. M. Marques, M. Labiche, N.A. Orr, J. C. Angélique, L. Axelsson, B. Benoit, U. C. Bergmann, M. J. G. Borge, W.
N. Catford, S. P. G. Chappell, N. M. Clarke, G. Costa, N. Curtis, A. D'Arrigo, F. de Oliveira Santos, E. de Góes Brennand, O. Dorvaux, M. Freer, B. R. Fulton, G. Giardina, C. Gregorie, S. Grévy, D. Guillemaud-Mueller, F. Hanappe, B. Heusch, B. Jonson, C. Le Brun, S. Leenhardt, M. Lewitowicz, M. J. López, K. Markenroth, M. Motta, A. C. Mueller, T. Nilsson, A. Ninane, G. Nyman, I. Piqueras, K. Riisager, M. G. Saint Laurent, F. Sarazin, S. M. Singer, O. Sorlin and L. Stuttgé, Phys. Lett. B 476, 219 (2000).

[16] F. M. Marques, M. Labiche, N.A. Orr, J. C. Angélique, L. Axelsson, B. Benoit, U. C. Bergmann, M. J. G. Borge, W. N. Catford, S. P. G. Chappell, N. M. Clarke, G. Costa, N. Curtis, A. DArrigo, E. de Góes Brennand, F. de Oliveira Santos, O. Dorvaux, G. Fazio, M. Freer, B. R. Fulton, G. Giardina, S. Grévy, D. Guillemaud-Mueller, F. Hanappe, B. Heusch, B. Jonson, C. Le Brun, S. Leenhardt, M. Lewitowicz, M. J. López, K. Markenroth, A. C. Mueller, T. Nilsson, A. Ninane, G. Nyman, I. Piqueras, K. Riisager, M. G. Saint Laurent, F. Sarazin, S. M. Singer, O. Sorlin, and L. Stuttgé, Phys. Rev. C 64, 061301 (2001).

[17] G. Verde, D. A. Brown, P. Danielewicz, C. K. Gelbke, W. G. Lynch, and M. B. Tsang, Phys. Rev. C 65, 54609 (2002).

[18] G. Verde, P. Danielewicz, D. A. Brown, W. G. Lynch, C. K. Gelbke and M. B. Tsang, Phys. Rev. C 67, 34606 (2003).

[19] D. O. Handzy, W. Bauer, F. C. Daffin, S. J. Gaff, C. K. Gelbke, T. Glasmacher, E. Gualtieri, S. Hannuschke, M. J. Huang, G. J. Kunde, R. Lacey, T. Li, M. A. Lisa, W. J. Llope, W. G. Lynch, L. Martin, C. P. Montoya, R. Pak, G. F. Peaslee, S. Pratt, C. Schwarz, N. Stone, M. B. Tsang, A. M. Vander Molen, G. D. Westfall, J. Yee, and S. J. Yennello, Phys. Rev. Lett. 75, 2916 (1995).

[20] G. F. Bertsch and S. Das Gupta, Phys. Rep. 160, 189 (1988)

[21] J. Aichelin, Phys. Rep. 202, 233 (1991).

[22] L. W. Chen, V. Greco, C. M. Ko, and B. A. Li, Phys. Rev. C 68, 014605 (2003).

[23] Y. B. Wei, Y. G. Ma, W. Q. Shen, G. L. Ma, K. Wang, X. Z. Cai, C. Zhong, W. Guo, J. G. Chen, Phys. Lett. B 
586, 225 (2004).

[24] Y. B. Wei, Y. G. Ma, W. Q. Shen, G. L. Ma, K. Wang, X. Z. Cai, C. Zhong, W. Guo, J. G. Chen, D. Q. Fang, W. D. Tian, X. F. Zhou, J. Phys. G 30, 2019 (2004).

[25] S.E. Koonin, Phys. Lett. B 70, 43 (1977).

[26] S. Pratt, Phys. Rev. Lett 53, 1219 (1984).

[27] S. Pratt, M. B. Tsang, Phys. Rev. C 36, 2390 (1987).

[28] S. Pratt, Nucl. Phys. A 566, 103c (1994).

[29] J. Aichelin, A. Rosenhauer, G. Peilert, H. Stöcker and W. Greiner, Phys. Rev. Lett. 58, 1926 (1987).

[30] G. F. Westfall, W. Bauer, D. Craig, M. Cronqvist, E. Gaultieri, S. Hannuschke, D. Klakow, T. Li, T. Reposeur, A. M. Vander Molen, W. K. Wilson, J. S. Winfield, J. Yee, S. J. Yennello, R. Lacey, A. Elmaani, J. Lauret, A. Nadasen, E. Norbeck, Phys. Rev. Lett. 71, 1986 (1993).

[31] D. Klakow, G. Welke, and W. Bauer, Phys. Rev. C 48, 1982 (1993).

[32] Y. G. Ma, W. Q. Shen, J. Feng, Y. Q. Ma, Phys. Rev. C 48, R1492 (1993); Y. G. Ma and W. Q. Shen, Phys. Rev. C 51, 3256 (1995).

[33] K. Chen, Z. Fraenkel, G. Friedlander, J. R. Grover, J. M. Miller, and Y. Shimamoto, Phys. Rev. 166, 949 (1968).
[34] R. Ghetti, J. Helgesson, N. Colonna, B. Jakobsson, A. Anzalone, V. Bellini, L. Carlén, S. Cavallaro, L. Celano, E. De Filippo, G. DErasmo, D. Di Santo, E. M. Fiore, A. Fokin, M. Geraci, F. Giustolisi, A. Kuznetsov, G. Lanzanó, D. Mahboub, S. Marrone, F. Merchez, J. Martensson, F. Palazzolo, M. Palomba, A. Pantaleo, V. Paticchio, G. Riera, M. L. Sperduto, C. Sutera, G. Tagliente, M. Urrata, and L. Westerberg, Phys. Rev. C 64, 017602 (2001).

[35] R. Ghetti, J. Helgeson, V. Avdeichikov, P. Golubev, B. Jakobsson, N. Colonna, G. Tagliente, S. Kopecky, V. L. Kravchuk, H. W. Wilschut, E. W. Anderson, P. NadelTuronski, L. Westerberg, V. Bellini, M. L. Sperduto, and C. Sutera, Phys. Rev. Lett. 91, 092701 (2003).

[36] Y. G. Ma, Q. M. Su, W. Q. Shen, D. D. Han, J. S. Wang, X. Z. Cai, D. Q. Fang, H. Y. Zhang, Phys. Rev. C 60, 24607 (1999).

[37] P. Danielewicz, R. Lacey and W. G. Lynch, Science 298, 1592 (2002).

[38] H. Fuchs and K. Mohing, Rep. Prog. Phys. 57, 231 ( 1994) and refernces therein. 